Revue Sciences/Lettres

Sciences / Lettres

4 | 2016

Baba Yaga en chair et en os

\title{
Baba Cloantza, la Yaga édentée du folklore roumain
}

Simona Ferent

\section{(2) OpenEdition \\ Journals}

Édition électronique

URL : http://journals.openedition.org/rsl/993

DOI : $10.4000 /$ rsl.993

ISSN : 2271-6246

Éditeur

Éditions Rue d'Ulm

Référence électronique

Simona Ferent, «Baba Cloantza, la Yaga édentée du folklore roumain », Revue Sciences/Lettres [En ligne], 4 | 2016, mis en ligne le 16 janvier 2016, consulté le 01 mai 2019. URL : http:// journals.openedition.org/rsl/993 ; DOI : 10.4000/rsl.993

Ce document a été généré automatiquement le 1 mai 2019.

(c) Revue Sciences/Lettres 


\title{
Baba Cloantza, la Yaga édentée du folklore roumain
}

\author{
Simona Ferent
}

1 Tout en intégrant la réflexion initiée par l'association LETAP en janvier 2015 intitulée «Baba Yaga et son isba, analyse pluridisciplinaire du personnage mythique des contes populaires russes » nous vous proposons l'exploration d'une autre baba, Baba CloanŢa personnage central des contes roumains. Pour une traduction phonétique la plus fidèle possible, nous avons opté pour la forme « Cloantza ». Puisant dans le folklore des Carpates les contes, vieux ou récents, mettent en scène le personnage d'une vieille sorcière facilement reconnaissable grâce à certains traits particuliers. Avant de procéder à une analyse des séquences livresques, notons que l'invocation du nom de Baba Cloantza suggère pour tout lecteur roumain une représentation corporelle singulière, dont le symbole qui se détache est l'absence de dents. Le terme de cloanţa prononcé [kloantza] signifie « une femme vieille, laide et sans dents ${ }^{1}$ ». Dans l'univers des contes, il résume le syntagme de méchante sorcière. Par ailleurs, le même mot est employé dans le langage familier pour désigner de manière péjorative "la bouche ${ }^{2}$ », comme organe d'expression (qu'on pourrait traduire vulgairement comme «la baba grande gueule»). L'appellation populaire insiste sur sa vieillesse (marque d'une sagesse inquiétante), mais aussi sur sa fonction de vecteur d'une parole. Cette parole est d'autant plus puissante que la baba serait impuissante à mordre.

2 À cette symbolique particulière, l'imaginaire populaire superpose un deuxième sens qui est celui d'une vieille femme vivant en périphérie du village, souvent dans la forêt, d'une apparence effrayante et pratiquant des rituels magiques. Le terme «baba » exprime, de manière plus générale, la vieille sage, ou plus particulièrement la sorcière à laquelle le village attribue tous les pouvoirs traditionnels allant du sort d'amour, à la divination, la maîtrise des éléments météorologiques et jusqu'aux pouvoirs de guérison.

3 À partir de l'étude de cas représentatifs, notre réflexion insistera sur deux dimensions $d u$ personnage : d'un côté, l'ambiguïté de la figure de la baba, à la fois créature démoniaque (image d'une altérité archaïque) et guide spirituel (la sorcière dont les pouvoirs magiques 
aident le voyageur, la jeune femme en mal d'amour ou le héros dans son combat avec des forces surnaturelles); de l'autre côté, la richesse et la diversité des figures artistiques de la baba Cloantza et ses avatars. Notre analyse propose ainsi une typologie des différentes représentations de la baba Cloantza qui espère susciter l'intérêt du lecteur français pour la mythologie populaire roumaine qui abrite d'autres créatures tout aussi ensorcelantes que les bien connus vampires.

4 Le corpus que nous avons choisi de présenter ici comporte un ensemble varié de textes appartenant à des auteurs roumains ayant puisé dans la matière folklorique afin de donner une seconde vie aux mythes. Pour Vasile Alecsandri (1821-1890), immense personnalité littéraire dont les poèmes et les pièces de théâtre constituent des classiques pour les élèves roumains, la collecte des ballades et autres productions orales traditionnelles n'est pas uniquement un travail de mémoire nécessaire mais surtout, dans le contexte historique, l'affirmation d'une identité culturelle roumaine. Dans les ballades populaires qu'il nous a léguées mais aussi dans ses réécritures poétiques (dont la plus réussie porte le nom de "Baba Cloantza »), la sorcière occupe souvent le devant de la scène pour jouer son rôle de prophète, de guérisseuse ou de démon. Si le barde amoureux du folklore de la Transylvanie, George Coşbuc (1866-1918) reprend l'image de la baba réinvestissant le mythe d'un éternel féminin aux origines daces, d'autres poètes comme Tudor Arghezi (1880-1967) au siècle dernier et Gelu Vlaşin (1966-) en 2000 nous proposent des versions modernes et une baba Cloantza réduite à un personnage caricatural. Sa bouffonnerie théâtrale se retrouve déjà représentée dans la première opérette roumaine composée en 1848 intitulée «Baba Hârca ». Mais puisque Baba Cloantza puise ses sources dans l'univers des contes, nous examinerons ses manifestations dans plusieurs histoires célèbres du grand folkloriste Petre Ispirescu (1830-1887) et du mémorialiste du monde rural Ion Creanga (1837-1889). Baba Cloantza y surgira comme une composante incontournable du parcours initiatique du héros légendaire.

\section{Baba Cloantza, oracle du village}

Dans la mythologie populaire roumaine le Sburător incarne la séduction maléfique par une créature fantastique qui hante la nuit le sommeil des femmes. Lorsqu'en 1843 Vasile Alecsandri met en vers ce mythe du vampire dans un poème d'inspiration folklorique

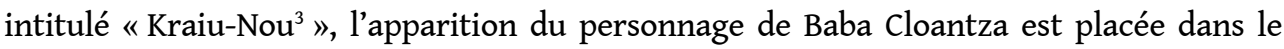
cadre d'une nature paysanne idyllique dominée par le symbole lunaire. La temporalité spécifique du calendrier populaire associe la manifestation de l'astre nocturne (le KraiuNou correspondant à la première phase de la lune) à un moment propice pour exprimer des vœux (d'amour pour la plupart) : « À cette heure du soir, où l'oiseau vole à son nid en jetant un cri plaintif comme un soupir ; / [...] Zamfira, triste et pensive, sortait de sa tente et fixait des regards humides de larmes sur la lune qui répandait sa blanche lumière sur le front de la jeune fille. ${ }^{4}$ "

6 Parmi les jeunes qui adressent leur invocation magique à la lune, le poète évoque Zamfira, prototype romantique de la beauté virginale de la paysanne roumaine, qui sous l'emprise de ses premières pulsions érotiques choisit d'ignorer la mise en garde de Baba Cloantza: après un acte divinatoire propre à la baba (qui consulte ses quarante et un grains de maïs), celle-ci lui conseille avec effroi de fuir « le bel étranger à la voix caressante ${ }^{5}$ ». La baba est représentée assise sur un monticule et le lecteur comprendra par la suite qu'il s'agit en fait de la tombe d'un être vampirique. Dans le poème la mention faite à Baba 
Cloantza suit la strophe décrivant les vieux sages du village qui prévoyaient un avenir heureux à la jeune fille. Mais Zamfira succombe aux charmes d'un mystérieux amant qui descend des ombres nocturnes et dévore peu à peu sa force vitale.

Trois jours après, le croissant s'effaça du ciel, et, comme lui, le bel étranger disparut. La pauvre fille s'assit au bord du chemin, et le regretta beaucoup et pleura beaucoup après lui.

Trois jours après, là-bas dans la vallée, il ne restait plus que son tombeau, et bien longtemps on entendait une voix plaintive passer dans le vent de la nuit et répéter avec douleur :

«Toi, qui vas gaiement sur la colline pour confier les secrets de ton âme au croissant de la lune, ô pauvre jeune fille, fuis à la tombée de la nuit, fuis le bel étranger à la voix caressante. $»^{6}$

Placée en marge de la communauté, la baba Cloantza joue ici entièrement son double rôle dans le sens où elle prévient et provoque le danger. Sa fonction d'oracle met en place les conditions d'une prophétie auto-réalisatrice. Ses paroles détiennent la formule à la fois de la peur et de la séduction qu'elle résume dans l'image d'une altérité totale (l'étranger est celui qui vient d'un autre monde, céleste et maléfique, un être démoniaque). Le personnage de la baba permet autant la mise en scène d'une voix rappelant une croyance populaire (celle qui associe les apparitions fantomatiques pendant le premier croissant de lune aux êtres maléfiques) que l'expression d'une morale archaïque de mise en garde sur la sexualité. Ce sont les manifestations magiques qui assurent la dimension pédagogique du conte.

\section{La baba guérisseuse}

8 La baba est également invoquée par l'homme désespéré par un amour qu'il en vient à maudire. La poésie populaire "Burueana de leac ${ }^{7}$ » (à traduire «la mauvaise herbe qui soigne ${ }^{8}$ ", titre révélateur) est une illustration des prières qui peuvent être adressées par un paysan à la baba du village. Sous l'emprise d'une passion qui ressemble fortement à une possession maléfique (à travers surtout les symboles du soleil qui s'éteint et du tombeau qui l'appelle), il déclame ses vers afin de solliciter les pouvoirs magiques de la mama Ileana, seuls capables d'éteindre le feu du cœur. Comme une note explicative du folkloriste V. Alecsandri le souligne, la mère Ileana n'est qu'un des avatars de la baba. Celle-ci remplit un statut de guérisseuse dans les villages roumains. Elle soigne à l'aide de plantes et de paroles magiques en fonction de chaque période du calendrier populaire, véritable croisement de fêtes religieuses et païennes. Au cœur de cette ambiguïté de l'imaginaire populaire, la baba en fait ressortir la dimension paradoxale en invoquant à la fois des objets religieux (des icônes), des lieux sacrés (l'église du village), des personnages bibliques (un chant faisant référence à la Sainte Marie mère de Jésus ainsi qu'à Dieu), au sein d'une effervescence de rituels païens (fleurs, mauvaises herbes, une eau investie de pouvoirs magiques, trois baguettes de coudrier, des paroles qualifiées de sorcellerie).

\section{Baba Cloantza et ses caricatures}

Puisant dans le réservoir mythique, les poètes roumains réinvestissent cette image de la guérisseuse. Dans un recueil de vers pour enfants paru en 1948, Tudor Arghezi imagine une baba ${ }^{9}$ qui fait des miracles dans le village : elle connaît tous les sorts d'amour, elle peut unir ou séparer les amants, elle détient les remèdes à toutes les douleurs (du mal des 
dents au mal du cœur). Le poète crayonne une caricature de sorcière bossue qui, à l'aide de " deux charbons et trois mensonges", peut soigner tout le monde sauf les pauvres, car pentru om sàrac / n-are leac ${ }^{10}$ (à traduire " pour le pauvre / elle n'a pas de remède ». En roumain la distance ironique est renforcée grâce à la rime qui s'appuie sur deux termes clés : minuni / minciuni c'est-à-dire, « miracles / mensonges ».

La figure de la baba Cloantza surgit dans les vers du poète contemporain Gelu Vlaşin comme matérialisation d'un fantasme. Entre l'ivresse et le délire, le poète erre dans les rues de la ville en quête d'un sort de fortune à dépenser dans le premier bordel. La référence à la Cloantza revêt ici le sens d'une réminiscence de l'inconscient collectif qui vient s'immiscer dans le réel « dilaté » déjà par Dionysos et investi par un ensemble de symboles à ample connotation psychologique (le nain, le cirque, "le bar à putes", des araignées, etc.) : «comme dans un conte pour les cons / je cherche la baba cloantza / pour qu'elle me jette des sorts / d'argent » (notre traduction des vers ca-ntr-o poveste cu prosti / caut baba cloanŢa / să-mi descânte vrăji / cu bani mulţi $i^{11}$ ).

11 Un autre avatar de la Cloantza, Baba Hârca, apparaît quant à elle dans les contes sous la forme d'une vieille sorcière vivant recluse dans les grottes au fond des bois (par peur des humains) et qui utilise des crânes humains ou d'animaux dans ses rituels magiques. Epithète dépréciatif qualifiant une "vieille femme, laide et méchante ", en roumain, le terme de hârca ${ }^{12}$ signifie aussi « crâne ». Dans les contes retranscrits ou composés, par les écrivains et folkloristes roumains nous observons une souplesse dans la dénomination du personnage. Les appellations Baba Cloantza et Baba Hârca deviennent synonymes au sein d'une même histoire ou alternent en fonction des variantes du même conte.

C'est sous le nom de Baba Hârca que la sorcière joue son rôle magistral, dans la première opérette roumaine, composée par Matei Millo et Alexandru Flechtenmacher en 1848 et transposée par le musicien slovène Davorin Jenko sur des territoires serbes. «Baba Hârca » affiche le sous-titre "opérette-sorcellerie en deux actes et trois tableaux ${ }^{13}$ ". Matei Millo y interprète lui-même le rôle de la Hârca, en campant un personnage de gitane traité de façon comique.

\section{La sorcière et la magie du rituel}

13 Nous recomposons aujourd'hui les traits de celle qui ne représente qu'un personnage légendaire quelque part entre la nostalgie de l'enfance et le souvenir livresque d'un espace pastoral résolument disparu. Son altérité nous paraît radicale. Elle ne constitue pour nous qu'un être de fiction. Mais lorsque l'écrivain et folkloriste V. Alecsandri, enregistre des productions populaires, comme Cucul si Turturica ${ }^{14}$ [Le Coucou et la Tourterelle], il insiste dans les notes explicatives sur la description soignée des superstitions populaires. Pour nombre de paysans roumains, le pouvoir de la baba est bien réel. Ainsi, à travers le dialogue entre la tourterelle (symbole de l'amour angélique) et le coucou (oiseau mystérieux, voire dangereux car fils d'une méchante sorcière), l'allégorie transcrit une réalité qui est celle de la sorcellerie rurale. Conservée dans la langue parlée par une somme de proverbes associant la sorcière au démoniaque, la baba incarne au sein du village une entité surnaturelle. Baba Cloantza est ainsi un être dont la différence est acceptée et intégrée par la communauté. Placée en intermédiaire entre le monde des vivants et un au-delà fantastique, la sorcière agit comme un élément catalyseur indispensable au sein du rituel magique. Guérisseuse exercée, la baba «prend 
sur elle le mal ", devient une véritable bouche qui aspire les angoisses de l'autre qu'elle expulse ensuite en leur donnant une forme symbolique. Le rituel est une mise en acte d'une projection fantasmagorique et il faut insister sur la dimension performative de la parole de la baba. Rappelant que le mot «cloantza » abrite la métaphore de la bouche édentée, il nous semble pertinent d'envisager le rituel magique de la sorcière comme un acte de digestion: les angoisses des paysans en fournissent l'aliment et les paroles de Baba Cloantza la substance magique.

Sur le fond d'un hédonisme et d'un animisme profonds, le rituel et les paroles de la baba inspirent autant la peur qu'un certain fatalisme. Personnification d'un esprit joueur et bon vivant, le coucou chante son désir et sa détermination à user des moyens peu orthodoxes auxquels il aurait accès grâce à sa génitrice pour séduire et consommer sa passion. L'influence maléfique de la baba Cloantza dans la conquête amoureuse est à envisager sous le signe du jeu. Ainsi pour figurer le danger gisant dans les jeux de séduction, le choix des objets magiques que les babas manient dans leurs sorts révèle une dimension burlesque : pour leurs rituels d'ensorcellement des jeunes hommes un peu « distraits ", les sorcières utilisent des os de chauve-souris attrapées la veille de Noël et enterrées vivantes dans une fourmilière. Ce qui reste du squelette de la chauve-souris prendra la forme d'un crochet et d'une petite pelle. La Cloantza se sert du premier objet pour accrocher l'être qu'on désire, alors que le deuxième éloigne tous ceux qu'on n'aime pas.

L'imaginaire paysan témoigne d'une impressionnante richesse quant aux pouvoirs des babas, dont l'une des constantes majeures semble être l'élément aquatique. Sous les formes les plus variées, l'eau acquiert des pouvoirs surnaturels : la sorcière se sert de l'eau des rivières, elle arrive à maîtriser la pluie, jette des sorts à l'aide de l'eau bénite ou au contraire à l'aide d'une eau enchantée dite " vierge »; ainsi l'un des sorts classiques est celui de la cruche, l'ulcica, dont un proverbe roumain dit i-a fàcut cu ulcica signifiant « il/ elle a subi le sort de la cruche ${ }^{15}$ ». D'autres éléments souvent mentionnés - comme la baguette de coudrier, les grains de maïs, le batic traditionnel (le fichu traditionnel des paysannes) - renvoient à cet univers paysan au sein duquel l'objet du quotidien s'anime ou, sous l'emprise d'une formule magique, devient un instrument enchanté.

La communion avec la nature est totale, une nature animée. Le paysan chante sa doïna (une oraison populaire à forte tonalité mélancolique) à son esprit-frère (ceci peut être un arbre, une fleur, un oiseau, un animal sauvage ou domestique ou souvent la forêt entière). Des créatures fabuleuses comme les ielele (les sirènes des bois) peuplent les montagnes et les collines. La croyance populaire veut que, sous l'influence d'un maléfice, un jeune homme puisse se retrouver à errer dans les plaines sur un bâton ensorcelé suivant la voix d'une Cloantza. Le folkloriste enregistre le témoignage de paysans convaincus d'avoir vus ces malheureux voyageurs transpercer le ciel comme des flèches. On apprendra aussi que seul un couteau planté dans la terre peut arrêter leur errance.

Il nous paraît important d'insister sur le poids de la récitation au sein de la formule magique. La baba accompagne ses gestes d'une invocation que le roumain appelle descântec, terme qui possède un double sens, celui d'une parole, chantée ou récitée, qui peut soit ensorceler et soit désensorceler. Ces invocations sont très nombreuses et répondent à une panoplie de situations, allant de la morsure d'un serpent jusqu'à une certaine peur de la solitude. Celle-ci est exprimée en roumain par la crainte du urât; le terme de urât se traduit à la fois par l'adjectif «laid» mais il résume également une angoisse difficilement traduisible qui concentre le sentiment de l'abandon et la peur de 
l'autre - si nous devions transposer cela au lecteur français nous utiliserions l'image d'une personne se retrouvant seule dans une maison hantée ${ }^{16}$. Baba Cloantza, personnage des fictions pour enfants, reprend donc l'ensemble des caractéristiques de la vieille sorcière $\mathrm{du}$ village dont le rôle principal consiste à offrir une somme de solutions magiques en réponse aux besoins réels des paysans. Il s'agit bien évidemment ici d'un village roumain archaïque baignant dans une réalité mythique que l'on retrouve dans les contes.

\section{Un archétype féminin protéiforme}

À partir de la matrice de la vieille prêtresse, initiée aux secrets de la nature, qui concentre une énergie originaire (la sorcière investissant des figures mythologiques comme Gaïa, Maya, Circé, Déméter, Isis, Lilith), la baba propose aussi l'image archétypale de la MèreTerrible Sorcière. Le principe de l'éternel féminin prend alors une dimension inquiétante. Sa capacité à engendrer des humains dotés de capacités physiques hors du commun est un élément constant de la mythologie populaire. Dans une évocation apologétique intitulée Atque nos $!^{17}$, le poète George Cosbuc n'oublie pas de mentionner le personnage de la baba sous cet aspect de génitrice du courageux jeune homme qui selon la formule roumaine consacrée "pousse en un an comme d'autres en dix ». Il fait l'éloge de cette alchimie magico-religieuse qui a donné naissance à une riche galerie de figures de l'universel féminin : la baba Dochia aux origines daces, la Mama-Noptii (la Mère de la Nuit) entourée de créatures vampiriques, ou les Saintes Mardi, Vendredi, Mercredi et Jeudi comme des fantaisies païennes, véritables métamorphoses des déités du panthéon grec (Mars, Zeus, Venus ou Mercure).

La critique souligne la présence de la baba dans les contes comme manifestation d'une force élémentaire incontrôlable. Ainsi, dans un de ses célèbres contes intitulé « Le Conte du cochon » [Povestea porcului ${ }^{18}$ ], l'écrivain Ion Creangă, met en scène la figure de la baba sous trois formes : premièrement, la très vieille paysanne qui, désespérant de sa stérilité, adopte un cochon qu'elle élève comme son fils (celui qui se révélera être le Prince charmant en proie à un sort); deuxièmement, les Trois Saintes - Mercredi, Vendredi et Dimanche - les sorcières qui guident l'héroïne dans son voyage initiatique à la recherche de son mari (c'est-à-dire à la fois le cochon et Prince charmant ensorcelé qu'elle avait épousé et qu'elle avait perdu en jetant au feu sa peau / son habit de cochon); et troisièmement, «l'Édentée-baba-Cloantza » qui garde le Prince charmant sous son emprise à l'aide de ses pouvoirs maléfiques. En déjouant les plans de la Cloantza et retrouvant sa bien-aimée, le héros délivre celle-ci du sort qui l'empêchait d'accoucher depuis quatre ans.

Sur la toile de fond d'une nature inquiétante, le conte du cochon semble hanté par l'idée d'une procréation maudite illustrée à travers trois manifestations : la stérilité, le sort qui empêche l'accouchement et le fait d'accoucher d'un démon. L'héroïne, enceinte d'un enfant qui ne peut naître, traverse un cadre fantastique peuplé par des dragons, des loutres à vingt-quatre têtes et d'autres créatures terrifiantes, mais surtout un monde défini comme le règne de la cupidité, de la ruse et de la méchanceté. L'image de la vilaine sorcière est ici construite en antithèse avec la jeune princesse naïve. Le conte l'identifie comme la Hârca (insistant ainsi sur sa vieillesse, sa fourberie, et sa laideur) mais surtout par le nom de Talpa Iadului ( La Taupe du Diable », n. t.). Ce personnage constitue l'un des êtres les plus maléfiques de la mythologie populaire roumaine car il est considéré comme 
la mère des démons. Elle se distingue par son intelligence et sa traitrise. La punition finale de celle-ci attachée à la queue d'un cheval réétablit l'élément comique qui ouvre d'ailleurs l'histoire, empêchant le conte de sombrer dans une morale qui se prête peu à l'ambiance fabuleuse créée.

21 Ce schéma initiatique de la fille cadette du roi se retrouve dans de nombreux contes. Le grand passionné de folklore roumain, Petre Ispirescu publie en 1876 «Le Cochon Ensorcelé » [Porcul cel Fermecat ${ }^{19}$ ] une histoire racontée par sa mère et qui reprend l'image antithétique mentionnée: Baba Cloantza (mère de dragons) et la jeune femme qui découvre à ses dépens les pouvoirs magiques et surtout les ruses de la méchante sorcière. Ici la baba personnifie une épreuve du Destin que l'héroïne doit surmonter pour parvenir au bonheur (symbolisé par le mariage d'amour). L'histoire décrit les aventures de la fille cadette d'un roi qui, suivant ses deux sœurs, entre dans la seule chambre du château à laquelle le père leur avait interdit l'accès. Les princesses y retrouvent un livre-oracle qui prédit des mariages princiers aux grandes sœurs, la cadette devra quant à elle épouser un cochon. Lisant le malheur sur le visage de sa fille, le vieux roi comprend la transgression. La prophétie se réalise et la princesse quitte la demeure paternelle pour suivre un cochon à la voix si humainement belle que le père soupçonne un terrible maléfice. La princesse apprend à aimer son étrange époux qui, tous les soirs, enlève la peau de cochon pour redevenir un homme. Mais lorsque la jeune mariée commence à s'habituer à ce train de vie peu commun (au point de tomber enceinte), la sorcière baba Cloantza «croise son chemin » et lui explique comment procéder pour chasser le sort. Sans se méfier de la sorcière, la jeune femme essaie d'utiliser la corde ensorcelée donnée par la baba pour attacher le pied gauche de son mari au lit. Mais la corde se casse et son époux se réveille pour lui apprendre que sans ce geste irréfléchi et précipité il aurait été délivré du sortilège trois jours plus tard. Le prince ensorcelé disparait et sa jeune épouse part à sa recherche, son enfant dans les bras. Traversant des contrées hostiles, la princesse roumaine trouve abri, conseil et objets magiques dans la maison de la Lune et de ses sœurs, la mère du Soleil et la mère du Vent. Arrivée devant l'étrange maison du prince ensorcelé, la jeune femme fait preuve d'intelligence et d'une extrême détermination : elle s'improvise une échelle avec les objets enchantés reçus (rien que des os de poulets) et va jusqu'à se couper le petit doigt pour s'en servir en guise de dernière marche. Les retrouvailles avec son mari permettent d'éclaircir les zones d'ombre de l'histoire, notamment le maléfice jeté par la Cloantza, mère d'un dragon tué par le prince lors d'une guerre. Avant le banquet final et la fin heureuse, la traversée du désert s'est imposée à la jeune femme qui a voyagé au-delà des frontières du monde réel. La transgression de la loi $\mathrm{du}$ père constitue le moteur déclencheur du parcours initiatique, alors que la baba Cloantza personnifie, dans ce contexte, l'agent du Mal à expier.

La même idée de la faute envers le père constitue le déclencheur du célèbre conte russe recueilli par Alexandre Afanassiev, La Plume de Finist-Fier-Faucon, qui donne son titre à un volume traduit en roumain Pana lui Finist Soimanul ${ }^{20}$. On y suit le même parcours de la jeune cadette qui cache à son père l'existence d'un amoureux qui lui rend visite magiquement chaque nuit. Par la jalousie de ses sœurs, cette romance sera tragiquement interrompue et la jeune fille devra quitter le foyer paternel pour traverser nombre d'épreuves afin de retrouver son amant. Lors de son périple, elle rencontrera la baba Cloantza ${ }^{21}$ à trois reprises sous trois formes différentes, laquelle lui servira à chaque fois de guide et d'alliée en lui fournissant les artefacts nécessaires à l'accomplissement de sa 
quête. Ces derniers, offerts par la petite, la moyenne, puis la grande baba, évoquent à la fois les Parques et le fil d'Ariane.

Le folkloriste Petre Ispirescu nous donne l'occasion de découvrir la baba Cloantza également comme mère d'un dragon que le jeune prince doit vaincre afin de délivrer une princesse esclave. Dans le conte intitulé «Histoire paysanne » [Poveste Tărănească ${ }^{22}$ ] le personnage de la Cloantza est placé au milieu d'un monde démoniaque dont le signe le plus manifeste consiste en une rangée de têtes empalées tout autour de sa cour. Plongé dans le monde du conte établi par le dispositif mythique du " Il était une fois », le lecteur retrouvera chez cette Cloantza une caractéristique similaire à celle du sorcier vaudou car elle puise sa force et son immortalité en avalant des esprits qu'elle garde prisonniers dans un tonneau. La menace principale qui plane d'ailleurs sur le royaume est celle des créatures vampiriques cherchant à s'emparer de l'âme du vieux roi.

\section{Baba Cloantza, une sorcière diabolique}

Baba-i calul dracului, (en traduction littérale « la Baba est le cheval du diable », traduction littéraire " Vieille sorcière, coursier de $\left.\operatorname{Satan}^{23} »\right)$, dit un ancien dicton roumain cité par V. Alecsandri en exergue d'un poème qu'il intitule Baba Cloanţa (écrit en 1842), travail d'inspiration folklorique qu'il qualifie lui-même comme une des ses meilleures improvisations ${ }^{24}$. La formule poétique reprend l'oralité du style populaire. Le lexique spécifique surchargé d'argot déplace la figure ambiguë de la baba vers une construction de sorcière shakespearienne. Ici c'est Cloantza elle-même qui semble possédée par un désir incontrôlé pour "le plus beau des jeunes garçons", désir qui s'empare d'elle dans une convulsion viscérale et résonnante : «Elle file, la vieille édentée; elle file en faisant claquer ses mâchoires et ses doigts; le fuseau de sa quenouille tourne rapidement en bourdonnant dans l'air ${ }^{25}$."

À travers une progression hyperbolique, le lecteur est transporté dans un cadre certes rural mais plongé entièrement dans une nuit infernale: dans le ciel nocturne des fantômes maléfiques peuplent les nuages, l'étang semble ensorcelé alors que des serpents rampent parmi les fleurs et «sur la branche élevée d'un arbre reluisent soudain deux yeux ennemis ${ }^{26}$ ». Dans cet éden dévoyé, sous le signe de la "lune pâle et blonde », la Cloantza invoque divers démons (Fiară-Verde, Sânge-Rosu, Hraconit), tout en proférant des menaces à l'encontre du jeune homme qui résisterait à ses charmes.

Que ses yeux tournent dans leurs orbites; que sa langue soit prise et que Satan, armé d'un fer brûlant, lui arrache le cœur de la poitrine pour le jeter dans les flammes éternelles. Que le monstre vert le poursuive (poursuit la Baba de plus en plus enragée, hystérique) tant qu'il y aura devant lui de la terre pour courir et de la lumière pour voir! Que les terribles esprits de la nuit, Hraconit et Sang-Rouge, viennent le torturer à leur tour jusqu'à l'aurore ${ }^{27}$.

Lorsque les démons invoqués (dont un vârcolac, un être vampirique qui, selon les croyances populaires, "mange le soleil et la lune ») échouent, la sorcière "gémit et pleure, elle a fini de filer, hélas! et son bien-aimé n'est pas venu ${ }^{28}$ ». La vieille Cloantza nomme alors Satan lui-même en lui proposant le pacte ultime « sans plus songer au péché mortel » : « viens accomplir mon vœu au prix de mon âme, que je te cède pour l'éternité ${ }^{29}$ ». Le pacte avec le diable déclenche un tourbillon de manifestations rappelant la démence et/ou la possession: elle court "follement ${ }^{30}$ ", saute, vole, elle crie des exorcismes. Décrite comme une "vieille échevelée ${ }^{31}$ », la «folle Kloantza » entourée de 
« milliers d'esprits infernaux » n'entend pas le « long éclat de rire ${ }^{32} »$ retentissant dans la forêt auquel un autre rire répond en écho. À deux pas de son «bien-aimé », la Cloantza voit son rêve basculer dans le cauchemar: le chant du coq réveille le village et les fantômes de la nuit se dissipent. Le tableau final se concentre ici encore sur l'élément aquatique : Satan se jette dans les profondeurs de l'étang aspirant également sa proie, la baba. La nature retrouve le calme et la sérénité mais le danger ne disparaît pas car les chuchotements d'une voix mélancolique appellent encore le voyageur «attardé [qui] passe en sifflant pendant la nuit au bord de cet étang ": « Viens à moi, mon brave chéri ; viens, je te chanterai pour toi la nuit de douces chansons, et je te soignerai comme une fleur, et je te préserverai par mes exorcismes du mauvais œil, des destinées cruelles et de la morsure des serpents ${ }^{33}$ ».

Comme la critique le souligne, l'œuvre permet deux pistes interprétatives : l'une qui examine cette ballade d'inspiration populaire comme une construction épique figurant l'amour malsain, inapproprié de la femme âgée pour le jeune homme ou, dans le langage $\mathrm{du}$ conte, le désir de la méchante sorcière pour le Prince charmant ${ }^{34}$; une deuxième perspective insiste sur la dimension humoristique au sens de "chicane» (donc, " chamaillerie, bagarre, querelle de mauvaise foi sur des détails de peu d'importance ») terme qui en roumain signifie « agacer, embêter quelqu'un avec des demandes infondées ${ }^{35}$ ». Il est important de rappeler alors la dualité de la baba à la fois personnage diabolique et esprit joueur. Opter pour une seule approche signifierait perdre une partie essentielle de son potentiel signifiant.

\section{La Cloantza, avatar de la Mort}

Dans un très ancien chant populaire, Holera ${ }^{36}$ (« Le Choléra », recueilli par V. Alecsandri en 1853) la Cloantza est figurée au prisme de la mythologie romaine des «Furies de l'enfer » représentées avec des serpents dans les cheveux. La métaphore de la sorcière (les cheveux ébouriffés, la peau asséchée sur son corps « envenimée ») révèle à nouveau le syncrétisme entre christianisme et paganisme: maniant des armes humaines et diaboliques, la Cloantza rappelle ainsi les représentations propres à la mythologie romaine des anges tenant dans la main droite un sabre de feu, signe d'une calamité naturelle à venir. Ici la Cloantza n'est autre que la mort (la maladie mortelle du choléra) qui surgit sur le chemin de l'insoucieux et bon vivant jeune Vâlcu. Cette fois aucune négociation n'est possible ${ }^{37}$. La Cloantza incarne la Faucheuse et il ne semble pas anodin qu'elle soit identifiée dans le chant par le terme « cloantza » employé seul. La disparition du nom « baba » enlève au personnage toute dimension humaine.

Partant à l'aventure dans l'univers du conte roumain nous avons pris pour guide Baba Cloantza qui s'est révélée porteuse de multiples visages et le médium de nombreuses voix. Et ces voix qui traversent la baba, ces voix qui soignent ou qui mutilent, qui font rire ou pleurer, qui jouent à l'oracle quand c'est la baba elle-même qui intriguera pour réaliser ses prophéties, se joignent à celle du conteur comme dans un chant choral et dévoilent à ceux qui les écoutent les pouvoirs de la mise en fiction. Lorsque le conteur narre les aventures de cette vieille femme édentée dont les pouvoirs pourraient se résumer à savoir mettre en récit les angoisses de ceux qui la consultent pour les diriger vers un avenir, c'est à lui-même et à son rôle qu'il fait référence. Privé d'un pouvoir réel, édenté lui aussi, le conteur dispose néanmoins de la magie des illusions qui nourrissent ou qui empoisonnent. Ainsi, lorsqu'il conte les histoires de la baba, c'est lui - même qui se 
raconte et l'on peut voir dans l'évolution de la figuration de la baba, l'évolution du rôle que peut se prêter le conteur: du rôle quasi mystique et thérapeutique de l'ancien temps au rôle subversif mais ironiquement inoffensif du contemporain.

\section{BIBLIOGRAPHIE}

Afanassiev, Alexandre, Pana lui Finist Soimanul : Basme fantastice rusesti [La plume de Finist-FierFaucon : contes fantastiques russes], trad. en roumain P. Stoicescu et A. Ivanov, Bucarest, éd. Ion Creanga, 1986.

Alecsandri, Vasile, Poésie roumane. Les Dö̈nas : poésies moldaves, trad. J. E. Voïnesco, Paris, Imprimerie de De Soye et Bouchet, 1853.

-, Poezii populare ale românilor, Bucarest, Éditions Minerva, notes Em. Gârleanu, coll. « Biblioteca Scriitorilor Români », 1908.

Arghezi, Tudor, Prisaca, Bucarest, éd. Ion Creanga, 1990.

Creangă, Ion, Povesti, Povestiri, Amintiri, Bucarest, éd. Eminescu, 1987.

Coteanu, Ion, Seche, Luiza et Seche, Mircea (dir.), DEX Dicţionarul explicativ al limbii române, Bucarest, Univers enciclopedic, 1996.

Ispirescu, Petre, Basmele românilor, Bucarest, Éditions eLiteratura, 2013.

-, Poveste tărănească, Povesti pentru Copii, 2015, http : //www.povesti-pentru-copii.com/petreispirescu/poveste-taraneasca.html, consulté en ligne le 15/05/2015.

Prisaca, Bucarest, éd. Ion Creanga, 1990, p. 40.

Millo, D.M., Baba Hârca : operetă vrajitorie în doua acte si trei tablouri, Bucarest, Tipografia C.A. Rosetti, 1851.

Nicolescu, Carmen, Daimonii in literatura românà, Craiova, éd. Universitaria, 2012.

Vlasin, Gelu, Atac de panică, Bucarest, éd. Soc. Culturala Noesis (édition en ligne, www.noesis.ro), 2000 / éd. Muzeul Literaturii Române, 2002.

\section{NOTES}

1. I. Coteanu et al. (dir.), DEX DicŢionarul explicativ al limbii române, p. 187.

2. Ibid., p. 187.

3. V. Alecsandri, « Kraiu-Nou ou la nouvelle lune », Les Doïnas : poésies moldaves, p. 34-37.

4. Ibid., p. 34.

5. Ibid., p. 36 .

6. Ibid., p. 37.

7. V. Alecsandri, « Burueana de leac », Poezii populare ale românilor, p. 174-175. 
8. Nom commun roumain composé de buruiana qui signifie "mauvaise herbe » et leac étant un synonyme populaire pour "remède». Le sens du syntagme renvoie à toute "plante sauvage médicinale ». DEX Dicţionarul explicativ al limbii române, Bucarest, op. cit., p. 120.

9. Nous faisons référence au poème «Baba-n sat » («La Baba dans le village », notre traduction) publié dans le recueil Prisaca, p. 40.

10. T. Arghezi, Prisaca, op. cit., p. 40

11. Nous citons en traduisant du poème « $15: 28$ », paru dans le recueil Atac de panică [Attaque de panique].

12. Le dictionnaire roumain renvoie à l'étymologie ukrainienne de hyrka. DEX Dicţionarul explicativ al limbii române, op. cit., 1996, p. 447.

13. D.M. Millo, Baba Hârca: operetă vrajitorie în doua acte si trei tablouri, [Baba Hârca: opérettesorcellerie en deux actes et trois tableaux].

14. V. Alecsandri, «Cucul si turturica », Poezii populare ale românilor, op. cit., p. 9-11.

15. En roumain le terme utilisé est «neîncepută » qui signifie «à laquelle on a pas encore goûté ", par extension, «à laquelle on a pas encore touché ». Voir les notes de V. Alecsandri, op. cit., p. 10.

16. Voir également le poème populaire «Urâtul » publié par V. Alecsandri dans Poezii populare ale românilor, op. cit., p. 152.

17. Le poème sera publié pour la première fois en 1886 dans La Tribuna, le principal journal de Cluj, Roumanie. Poème consulté en ligne http : //www.povesti-pentru-copii.com/poezii-pentrucopii/george-cosbuc/atque-nos.html, le 14/05/2015.

18. I. Creangă, « Povestea porcului », p. 38-52.

19. P. Ispirescu, «Porcul cel Fermecat », Basmele românilor, p. 66-81.

20. Pana lui Finist Soimanul: Basme fantastice rusesti [La plume de Finist-Fier-Faucon: contes fantastiques russes], p. 3-14.

21. Notons que dans cette version roumaine du conte d'Afanassiev, le terme de «Baba Yaga » est traduit par celui de "Baba Cloantza », une adaptation lexicale qui semble s'imposer devant un lecteur roumain moins sensible aux sonorités russes du nom de la sorcière.

22. Petre Ispirescu, Poveste tărănească.

23. La traduction appartient à J.E. Voinescu, in Poésie roumane. Les Doïnas : Poésies moldaves, de V. Alexandri, p. 19. La version française du poème s'intitule «La Vieille Kloantza»; le terme «baba » étant traduit soit par l'adjectif «vieille» soit par la formule «vieille sorcière » en fonction du contexte.

24. Les mots du poète sont cités par Carmen Nicolescu, dans sont travail sur le monde daïmonique dans la littérature roumaine, qui propose une analyse approfondie du poème. Daimonii in literatura românà, p. 75.

25. V. Alecsandri, «La Vieille Kloantza », in Poésie roumane. Les Doïnas : Poésies moldaves, op. cit., p. 20.

26. Ibid., p. 22.

27. Ibid., p. 20.

28. Ibid., p. 21.

29. Ibid., p. 22.

30. Ibid., p. 23.

31. Ibid., p. 22.

32. Ibid., p. 23.

33. Ibid., p. 24.

34. Dans son étude Carmen Nicolescu cite l'analyse de Serban Cioculescu, en l'opposant à l'interprétation de Alexandru Macedonski qui voit dans ce poème des situations de "chicane ». Daimonii in literatura românà, op. cit., p. 75, p. 79.

35. DEX Diç̧ionarul explicativ al limbii române, op. cit., p. 1055. 
36. V. Alecsandri, « Holera », Poezii populare ale românilor, op. cit., p. 27-28.

37. Nous rappelons qu'en échange d'avantages matériels, comme nous l'avons signalé dans d'autres contextes, la Baba Cloantza se mettait au service de celui ou celle qui faisait appel à elle et à ses pouvoirs.

\section{RÉSUMÉS}

Presque toute histoire inspirée du folklore des Carpates met en scène le personnage d'une vieille sorcière, d'une femme âgée vivant en périphérie du village, Baba CloanŢa [Kloantza]. Pour tout lecteur roumain bercé par les contes, ce nom suggère une représentation corporelle singulière, dont le symbole qui se détache, riche en termes d'analyse littéraire et mythique, est l'absence de dents. Notre réflexion s'attache à explorer l'ambiguïté de la figure de la Baba (guérisseuse, oracle, guide, démon, ou simple caricature) à travers un ensemble de textes représentatifs (contes, poèmes et opérette).

Nearly all the stories inspired by the Carpathian folklore feature the character of an old witch, an elderly woman living on the outskirts of the village, named Baba CloanŢa. For the Romanian reader, cradled by the ancient tales, this name suggests a singular (and symbolically charged) appearance marked by the absence of teeth. Our article examines the ambiguous nature of the Baba (as healer, oracle, spiritual guide, demon, or caricature) as presented in a number of edifying texts (tales, poems and an operetta).

\section{INDEX}

Mots-clés : réécriture du conte, folklore roumain, sorcellerie

Keywords : rewriting tales, Romanian folklore, witchcraft

\section{AUTEUR}

\section{SIMONA FERENT}

Simona Ferent, docteur ès lettres de l'Université de Limoges, est chercheuse indépendante et exerce le métier de professeur d'anglais à l'IFPM de Nanterre. Après un parcours littéraire, elle soutient en 2010 un mémoire de thèse intitulé Le JE et l'AUTRE, ou comment l'altérité répond à l'identité : questionnements chez Marthe Bibesco. Ses articles scientifiques portent un intérêt particulier à l'écriture de la mémoire et aux expressions de l'altérité dans les littératures française et francophone roumaine des $\mathrm{XIX}^{\mathrm{e}}$ et $\mathrm{XX}^{\mathrm{e}}$ siècles.

ferentsimona@yahoo.com 